\title{
Detecting Earnings Management. An Analysis of Credit Institutions' (Banks) Trading in Hungary
}

\author{
Endre Beretka \\ The Hague University of Applied Sciences, Den Haag, The Netherlands, The European Union \\ e.beretka@hhs.nl; beretka.endre@gmail.com
}

\begin{abstract}
This paper examines evidence of Earnings Management (EM) in annual financial reports of foreign and domestic credit institutions' trading in Hungary, an ex-communist country and a Central European economy, by applying an alternative approach, the Distribution of the Ratios method. Analyses were performed with 14 banking specific ratios for the period of 1999-2015, by applying Burgstahler and Dichev (1997), Degeorge et al. (1991) models, Kolmogorov-Smirnov, Monte-Carlo, Benchmark and Distributional tests. Primary findings confirm that (a) the Distribution of the Ratios method demonstrates that apart from significant evidence of EM presence, managers do not always manipulate the same variables, or one area of the financial statements, but at their discretion, choose different areas to engage EM and in different periods, and (b) Credit Institutions engaged in EM in the period Prior to and After the 2008 financial crisis. Additional Benchmark Analysis results present weak evidence of EM and should be read with caution; however, Benchmark comparison should not be excluded from research.
\end{abstract}

KEYWORDS: Earnings Management, Credit Institutions, Distributional Test, Ratios

\section{Introduction}

After the collapse of the communist regime in 1989, Hungary went through a political and economic transition. By 1999, credit institutions' (banks) have been privatized, and in 2004, Hungary becomes full member of the European Union (EU). Earlier papers of Szapáry (2001) and Várhegyi (2008) reported on consolidation, privatization, but no evidence of a research that examined Banks annual accounts, specifically in search for any evidence of EM. This paper set to fill this gap by testing annual statements of foreign and domestic credit institutions' (banks) trading in Hungary by applying a new approach. The rest of the paper is designed as follows: Summary of Literature Review, Methodology, Hypotheses and Data, Results, Discussion and Conclusion.

\section{Literature review}

Over the past 30 years, researchers have been mainly investigating non-financial company's annual accounts that might have led to accounting manipulations, thus to EM. Financial industry has not been as thoroughly investigated as non-financials due to strict rules that govern the financial industry and high financial penalties for cooking the books, sadly, the managers' incentive to engage in EM is not lower, but higher, see e.g., Shen and Chih (2005, 2696-2697). Since the '80's, studies investigated EM by applying accrual and/or the standard discontinuity models, see e.g., Beneish (1999), Shen and Chih (2005), or Gore, Pope and Singh (2007). Lately, an attempt to increase the power of tests were by Baber, Kang and Li (2011) and Dechow, Hutton et al. (2012) papers who attempted to incorporate reversal accruals into their testing models. However, the authors could not increase the testing power of more than $40 \%$, due to reversal accruals timing effect. One of the earliest studies which investigated Loan Loss Provisions (LLP), a specific accrual for financials, was by Beatty, Chamberlain and Magliolo (1995) who show weak evidence in favor of EM, whereas Beatty, Ke and Petroni (2002) present evidence of EM in publicly traded banks. From 2002 onwards, authors published papers showing evidence of EM within financials both at a cross-country and at a country level, see for example Beretka $(2016,12-98)$. Earlier studies examined EM by testing financials, mainly the LLP variable, and the non-financials data. These and earlier evidence suggest that accrual testing designs with few variables are weak in examining EM, see e.g., Dechow, Ge and Schrand's (2010) exploratory study that outlines the summary of strength and weaknesses of five accrual models linked to theories, which have been used to test evidence of EM. This paper proposes a new empirical approach to investigate EM by testing all available variables from the annual financial statements. This is discussed in the section bellow. 


\section{Hypotheses, Methodology and Data}

\section{Hypotheses}

This paper test total of 14 variables to investigate evidence of EM of foreign and domestic credit institutions' (Banks) annual accounts trading in Hungary. Two hypotheses were tested:

H0 $_{(\mathbf{a})}$ : Credit institutions' (Banks) in Hungary do not manage annual accounts.

H0 $\mathbf{b}_{(\mathbf{b})}$ : Credit institutions' (Banks) in Hungary do not manage annual accounts 'Prior to' and/or 'After' the 2008 financial crisis.

\section{Methodology}

Tests were performed with Burgstahler and Dichev (1997), Degeorge, Patel and Zeckhauser (1999) models, Kolmogorov-Smirnov, Monte-Carlo Method, benchmark and distributional tests. A total of 14 banking specific variables are tested on an annual basis. The two testing approaches are, see e.g., Beretka $(2016,129)$, the Distribution of Ratios Method, it has two testing designs, the EMI and the EM2 models that test Hypothesis $\boldsymbol{H O}_{(a)}$ : and $\boldsymbol{H O}_{(b)}$ : This paper calculates the 14 ratios by applying the EMI model, or,

$$
\left.\mathbf{E M 1}=\left(\mathrm{AO}_{\mathrm{i}}-\mathrm{EO}_{\mathrm{i}}\right) / \mathrm{SD}_{\mathrm{i}}\right)
$$

where, EMI is Earnings Management Model 1, and is equal to the Actual Observation (AO) in period (i) minus the Expected Observation (EO) in period (i). $\mathrm{SD}_{\mathrm{i}}=$ Standard Deviation, or, $\mathrm{SD}_{\mathrm{i}}=$ $\left[\mathrm{Np}_{\mathrm{i}}\left(1-\mathrm{p}_{\mathrm{i}}\right)+1 / 4 \mathrm{~N}\left(\mathrm{p}_{\mathrm{i}}-1+\mathrm{p}_{\mathrm{i}}+1\right)\left(1-\mathrm{p}_{\mathrm{i}}-1-\mathrm{p}_{\mathrm{i}}+1\right)\right]^{1 / 2}$, where, $\mathrm{SD}_{\mathrm{i}}=$ Standard Deviation of the difference in period (i); $p_{i}=$ probability of an observation in interval (i); $\mathrm{N}=$ number of total sample; $\mathrm{Np}_{\mathrm{i}}=$ total number of estimated Standard Deviation (SD) in interval (i); pi-1 = number in interval $\mathrm{i}-1$; $\mathrm{p}+1=$ number in interval $\mathrm{i}+1$. For additional explanation see Beretka $(2016,300-304)$. By applying the EM2 model, or,

$\mathbf{E M 2}=T_{\mathrm{n}}=\left[\Delta \mathrm{p}_{\mathrm{n}}-\operatorname{mean}(\Delta \mathrm{pi})\right] /$ s.d. $(\Delta \mathrm{pi})$

where, $i \in R, i \neq n$. $p_{i}$ is the ratio of the actual sample for year $i$ of banks years, $\Delta p n$ is the difference of $\mathrm{p}_{\mathrm{i}}-\mathrm{p}_{\mathrm{i}-1}$. Mean $(\Delta \mathrm{pi})$ is the average of $\Delta \mathrm{p}$ but excluding pi and s.d. ( $\left.\Delta \mathrm{pi}\right)$ is the standard deviation of $\Delta \mathrm{p}$, excluding $\Delta \mathrm{pi}$.

Test statistics with Kolmogorov-Smirnov (K-S) were performed, see, e.g., Massey (1951) for the Kolmogorov-Smirnov (K-S) test statistics, as well Lilliefors $(1967,399)$. K-S is applicable for ratio or interval data, a sample of $N$ observations: $\mathrm{D}=\max \mathrm{x} \in \mathrm{R}|\mathrm{F}(\mathrm{x})-\mathrm{F} 0(\mathrm{x})|$, where, $\mathrm{F}(\mathrm{x})$ is the cumulative normal distribution, and $\mathrm{F} 0(\mathrm{x})$ is the sample cumulative distribution, with $\mu=\bar{x}$ sample mean and $\sigma^{2}=\mathrm{s}^{2}$ sample variance with denominator $\mathrm{n}-1 . \mathrm{F}(\mathrm{x})=\mathrm{F} 0(\mathrm{x})$ for all $\mathrm{x}$ from $-\infty$ to $+\infty ; \mathrm{F}(\mathrm{x})$ $\neq \mathrm{F} 0(\mathrm{x})$ for at least one value of $(\mathrm{x})$. The K-S one sample test is non-parametric and distributionfree, an exact test, see, e.g., Panik $(2005,570)$. Additionally, benchmark analysis was calculated for each 14 ratio. Benchmark formula reads as:

$\overline{\mathbf{x}} \mathbf{i}, \mathbf{j}=\sum \mathrm{xi}, \mathrm{j} / \mathrm{Ni}, \mathbf{j}$

where, $\bar{x}_{j}=$ Is the Mean; $\sum x_{i, j}=$ The sum of all ' $x$ ' values; $N_{i, j}=$ The number of ' $x$ ' values, $i, j=$ Ratios and years respectively. Papers on benchmarking of non-financials are Kent and Routledge (2015); Sun and Rath (2012); Dechow, Ge and Schrand (2010); Crump and Teeguarden (2009);

Habib (2007); Dattakumar and Jagadeesh (2003); Yasin (2002). Testing sample on an annual basis with the EM1 and EM2 models for 'Prior to' and 'After' the 2008 financial crisis to test $\boldsymbol{H O}_{(\boldsymbol{b})}$ : with $\mathrm{K}-\mathrm{S}$ test statistics with 14 banking specific ratios. The tested 14 ratios for $\boldsymbol{H 0}_{(a, b)}$ : are: 
- Debt to Equity $(D T E)=$ Total Liabilities $_{(\mathrm{t})}$ divided by Equity $_{(\mathrm{t})}$.

- Equity to Loans $(E T L)=$ Average Equity $(\mathrm{t})_{\text {divided by } \text { Loans }_{(\mathrm{t})} \text {. Where: Average Equity }}^{(\mathrm{t})}=$ Equity $_{(\mathrm{t})}+$ Equity $\left._{(\mathrm{t}-1)}\right) / 2$

- Loans to Deposits Ratio $\left(\right.$ LTD) $=$ Loans $_{(\mathrm{t})}$ divided by Deposits $_{(\mathrm{t})}$.

- Loans to Total Assets $($ LTA $)=$ Loans $_{(\mathrm{t})}$ divided by Total Assets $(\mathrm{t})$.

- Gross Yield on Earning Assets $(G Y E A)=$ Tot. Interest Income $(\mathrm{t})$ divided by Tot. Earning Assets $(\mathrm{t})$.

- Rate Paid on Funds $(R P F)=$ Total Interest Expenses $(\mathrm{t})$ divided by Total Earning Assets $_{(\mathrm{t})}$.

- Sales Growth Index $(S G I)=$ Sales $_{(\mathrm{t})}$ divided by Sales $(\mathrm{t}-1)$.

- Interest (Sales) Receivables Index (IRI) $=$ [Accrued Interest receivables divided by sales $(\mathrm{t})$ ] divided by [Accrued Interest receivables divided by sales in receivables $(\mathrm{t}-1)]$.

- Gross Margin Index (GMI) = Gross Margin $_{(\mathrm{t}-1)}$ divided by Gross Margin $_{(\mathrm{t})}$. Where: Gross Margin $_{(\mathrm{t})}$

$=\left(\right.$ Total interest income $_{(\mathrm{t})}-$ Tot Int. expenses $\left.(\mathrm{t})\right)$ divided by Total Interest Income $_{(\mathrm{t})}$.

- Net interest Margin $($ NIM $)=$ Net Interest Income $_{(\mathrm{t})}$ divided by Average Earning Assets $_{(\mathrm{t}-1)}$. Where:

Net interest income $=$ Total Interest Income $(\mathrm{t})$ - Total Interest Expenses $_{(\mathrm{t})}$. Average Earning Assets correspond to all assets that earn income, assets.

- Profit Margin $($ PATM $)=$ Profit After Tax $(\text { PAT) })_{(\mathrm{t})}$ divided by Net Interest (sales) $)_{(\mathrm{t})}$.

- Return on Equity $(R O E)=$ Profit After Tax $(\mathrm{t})$ divided by Average Equity ${ }_{(\mathrm{t})}$.

- Return on Asset $(R O A)=$ Profit After Tax Tat $_{(\mathrm{t})}$ divided by Average Total Assets $(\mathrm{t})$.

Where: Average Total Assets $=\left(\right.$ Total $_{\text {Assets }}^{(t)}+$ Total Assets $\left._{(\mathrm{t}-1)}\right)$ divided by 2

- Equity to Total Assets $\left(\right.$ EtA) $=$ Equity $_{(\mathrm{t})}$ divided by Average Total Assets $_{(\mathrm{t})}$.

See, e.g., Beretka $(2016,142)$ for ratio calculations. Ratios are calculated for the same companies within a time frame, for example, company ' $z$ ' in year $(t)$ is calculated with the same company ' $z$ ' in year $(t-1)$ or in $(t-2)$.

\section{Data}

Audited data of publicly and privately owned foreign and domestic Credit Institutions - banks (operating as Joint-Stock Companies in Hungary) were obtained from the Central Bank of Hungary CBH and from the Hungarian Financial Supervisory Authority (HFSA). Since 1 October 2013, HFSA has become part of the $\mathrm{CBH}$ and operates under the umbrella of $\mathrm{CBH}, \mathrm{CBH} / \mathrm{HFSA}$ thereafter. Tested data is from 1999 to 2015 and consist of one listed (on the Budapest Stock Exchange; OTP Bank) and non-listed banks. For the period 1999-2015, CBH/HFSA published data annually and per Hungarian Accounting Standards (HAS); including for the listed OTP Bank. See HAS vs. IFRS differences in Table 3.1, Beretka (2016, 113-116). From 2017, CBH/HFSA introduces IFRS reporting, however, as per 2017 published data by the CBH/HFSA, only few banks switched from HAS to IFRS. For research purposes, CBH/HFSA gather individual companies (audited) annual accounts and create their own simplified version of the annual reports for each financial company, shown in Hungarian Forint (HUF). Hungary is not member of the European Monetary Union. Tested annual data consists of Profit and Loss Account and Balance Sheet. Cash Flow is not prepared nor published by CBH/HFSA. Number of individual banks per year is between 37 to 45 and per variable varies from 502 to 682 for the sample. This paper performed tests of Credit Institutions - banks annual accounts that were prepared under the HAS.

\section{Empirical Results}

\section{Statistical results of Ratios tested on the Annual basis with the EM1 model}

The Distribution of Ratio method is a hands on approach, based on statistical and distributional 
Table 1. One-Sample Kolmogorov-Smirnov (K-S) test run for all ratios on an annual basis with the EM1 model with $p=0.01$ Significance level

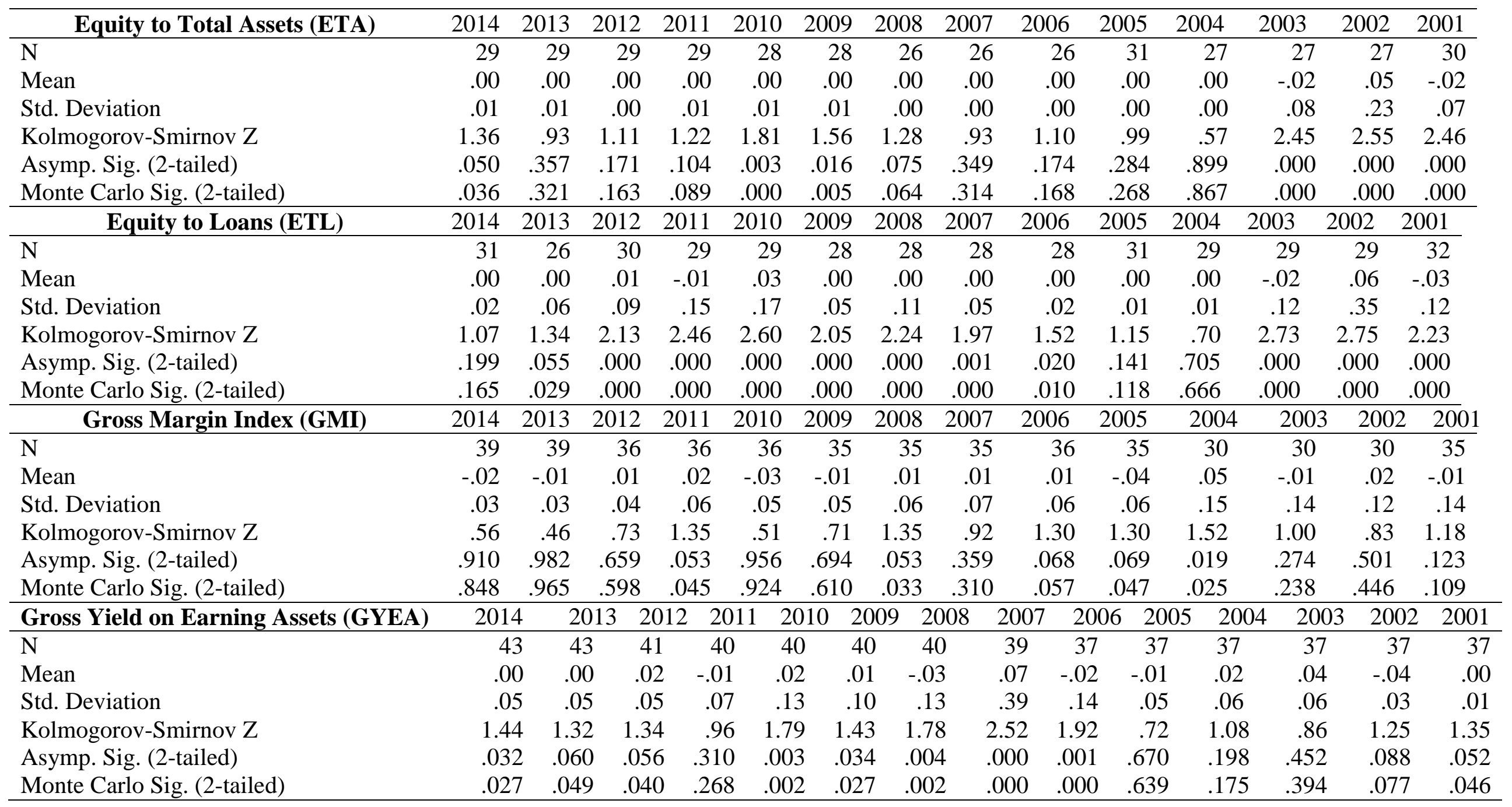

analysis of all computable ratios from the financial statements. Table 1 presents K-S test statistics with Monte with Monte Carlo simulation for each 14 ratios with 99\% Confidence Interval levels, as well as with $p=0.01$ Significance levels. Table 1. presents test outputs of each ratio for the period from 1999 to 2015, with low Mean and S.D. outputs, and negative and positive values. Test results for 
Table 1. Cont.

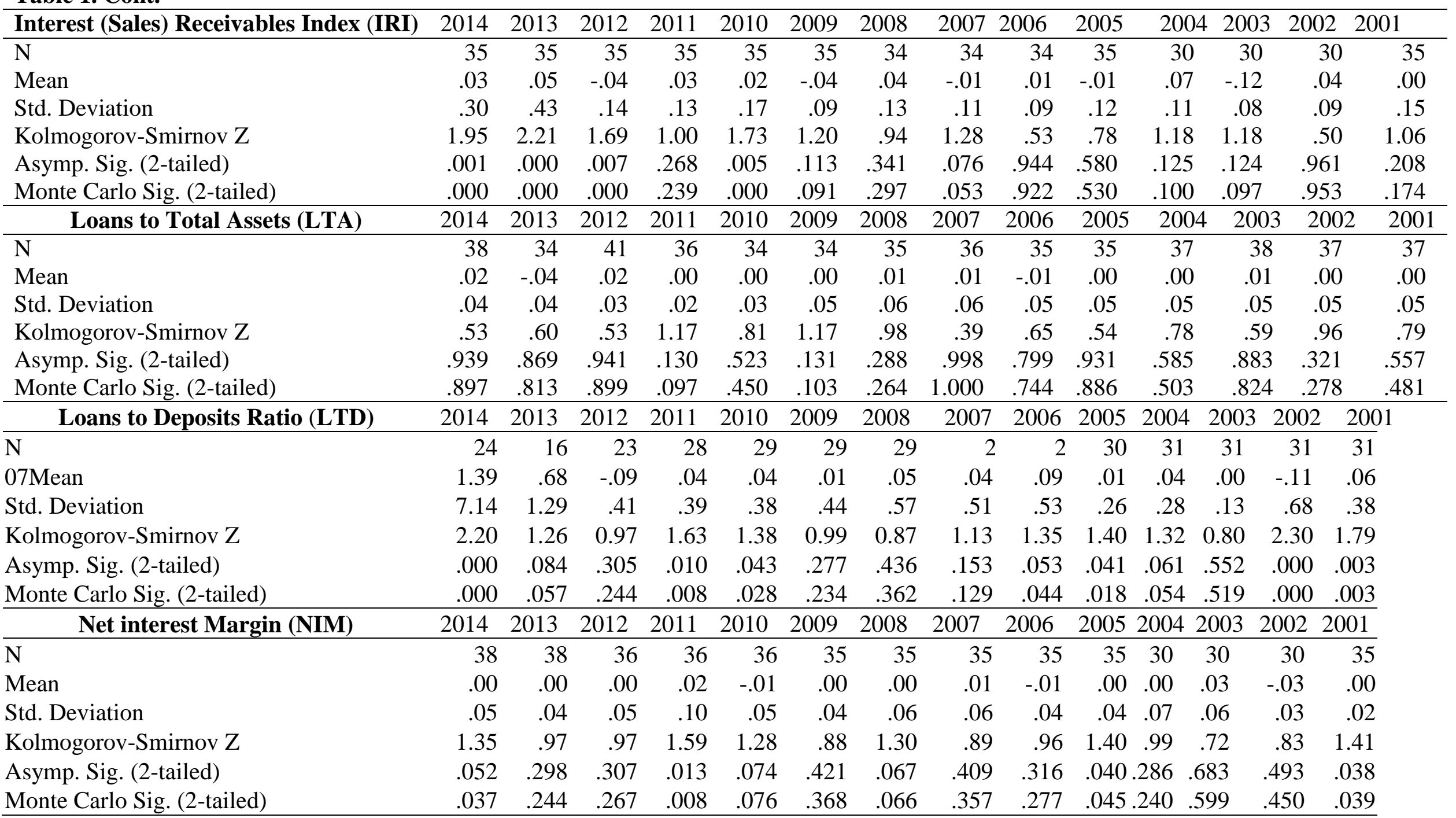

Asymp. Sig. and Monte Carlo Sig. statistically significant years for ETA are: 2001-2003 and 2010; for ETL: 2001-2003 and 2007-2012; for GMI, the results are statistically insignificant; for GYEA are: 2006-2008 and 2010; for IRI: 2010 and 2012-2014; for LTA, the results are statistically insignificant; for LTD are: 2001-2001, 2011, 2014; for NIM: 2011 for Monte Carlo Sig. only; for PATM: 2004-2005 and 2007; for ROA are: 
Table 1. Cont.

\begin{tabular}{|c|c|c|c|c|c|c|c|c|c|c|c|c|c|c|}
\hline Profit Margin (PATM) & 2014 & 2013 & 2012 & 2011 & 2010 & 2009 & 2008 & 2007 & 2006 & 2005 & 2004 & 2003 & 2002 & 2001 \\
\hline $\mathrm{N}$ & 39 & 41 & 41 & 38 & 38 & 38 & 38 & 40 & 36 & 37 & 34 & 34 & 34 & 33 \\
\hline Std. Deviation & .44 & .39 & .18 & .12 & .17 & .19 & .17 & .27 & .08 & .18 & .43 & .17 & .29 & .47 \\
\hline Asymp. Sig. (2-tailed) & .027 & .021 & .227 & .561 & .273 & .032 & .044 & .001 & .135 & .001 & .000 & .063 & .628 & .148 \\
\hline Monte Carlo Sig. (2-tailed) & .015 & .015 & .194 & .488 & .246 & .013 & .025 & .000 & .121 & .000 & .000 & .038 & .580 & .109 \\
\hline Return on Asset (ROA) & 2014 & 2013 & 2012 & 2011 & 2010 & 2009 & 2008 & 2007 & 2006 & 2005 & 2004 & 2003 & 2002 & 2001 \\
\hline Std. Dev & .01 & .01 & .01 & .01 & .01 & .01 & .00 & .01 & .01 & .00 & .00 & .00 & .01 & .00 \\
\hline Kolm & 1.02 & 1.10 & .87 & .78 & 1.03 & 1.06 & 1.05 & 1.76 & 1.83 & 1.03 & .79 & 1.04 & 1.87 & 1.69 \\
\hline Asymp. Sig. (2-tailed) & .253 & .175 & .438 & .583 & .240 & .215 & .218 & .004 & .003 & .239 & .568 & .234 & .002 & .007 \\
\hline Monte Carlo Sig. (2-tailed) & .220 & .134 & .396 & .535 & .191 & .185 & .187 & .002 & .000 & .217 & .500 & .195 & .000 & .002 \\
\hline Return on Equity (ROE) & 2014 & 2013 & 2012 & 2011 & 2010 & 2009 & 2008 & 2007 & 2006 & 2005 & 2004 & 2003 & 2002 & 2001 \\
\hline $\mathrm{N}$ & 21 & 15 & 18 & 18 & 16 & 18 & 17 & 18 & 17 & 20 & 21 & 20 & 21 & 25 \\
\hline Monte Carlo Sig. (2-tailed) & .011 & .558 & .381 & .038 & .068 & .023 & .158 & .008 & .294 & .049 & .230 & .196 & .068 & .004 \\
\hline Rate Paid on Funds (RPF) & 2014 & 2013 & 2012 & 2011 & 2010 & 2009 & 2008 & 2007 & 2006 & 2005 & 2004 & 2003 & 2002 & 2001 \\
\hline $\mathrm{N}$ & 37 & 38 & 37 & 37 & 37 & 38 & 38 & 38 & 37 & 37 & 37 & 35 & 35 & 35 \\
\hline Mean & .00 & .00 & .00 & .00 & .00 & .00 & .00 & -.02 & .11 & -.03 & .00 & .00 & .00 & .00 \\
\hline Std. D & .00 & .00 & .00 & .00 & .00 & .01 & .02 & .18 & .72 & .18 & .01 & .01 & .00 & .00 \\
\hline Kolmogorov-Smirnov 2 & .76 & .95 & .81 & .85 & .97 & 1.35 & 1.90 & 2.99 & 3.23 & 3.05 & 1.07 & 1.17 & 1.04 & 1.25 \\
\hline Asymp. Sig. (2-tailed) & .617 & .328 & .534 & .460 & .306 & .051 & .001 & .000 & .000 & .000 & .202 & .132 & .228 & .089 \\
\hline Monte Carlo Sig. (2-tailed) & .552 & .300 & .481 & .413 & .279 & .052 & .000 & .000 & .000 & .000 & .182 & .107 & .194 & .074 \\
\hline
\end{tabular}

2001-2002 and 2007; for ROE: 2001 and 2007; for RPF: 2005-2008; for SGI are: 2007-2009 and 2001 for Monte Carlo Sig only. 
Table 1. Cont.

\begin{tabular}{|c|c|c|c|c|c|c|c|c|c|c|c|c|c|c|}
\hline Sales & 2014 & 2013 & 2012 & 2011 & 2010 & 2009 & 2008 & 2007 & 2006 & 2005 & 2004 & 2003 & 2002 & 2001 \\
\hline $\mathrm{N}$ & 38 & 38 & 36 & 36 & 36 & 35 & 35 & 35 & 35 & 37 & 30 & 30 & 30 & 35 \\
\hline Mean & .01 & -.02 & .01 & .01 & -.04 & .04 & .00 & -.06 & .22 & -.13 & -.66 & 2.18 & -.58 & -.01 \\
\hline Std. & .07 & .04 & .06 & .06 & .09 & .20 & .23 & .32 & .20 & .09 & .39 & 1.59 & .58 & .30 \\
\hline Kol & 1.25 & .79 & .99 & 1.51 & 1.33 & 2.07 & 1.92 & 2.11 & 1. & .89 & .74 & 1.33 & 1.13 & 1.53 \\
\hline Asy & .088 & .559 & .285 & .021 & .058 & .000 & .001 & .000 & .117 & .405 & .642 & .058 & .158 & .018 \\
\hline Monte Carlo Sig. (2-tailed) & .045 & .502 & .265 & .004 & .031 & .000 & .000 & .000 & .097 & .356 & .591 & .045 & .105 & .008 \\
\hline
\end{tabular}

Finance managers' skills have become so sophisticated in manipulating accounting entries that evidence of EM may not emerge only in one section of the financial statements, but evidence may arise in multiple, or in all areas of financial statements and in diverse time frames. Table 1 presents this evidence, and as it was expected, not every year shows statistically significant test results, which suggests that EM is not present in every financial year for each ratio. This is in line with practise, as managers in order to hide they action, as well to avoid high financial penalities, they will engage in EM in different periods. The same is true for $95 \%$ Confidence Interval and at $p=0.05$ Significance levels for the EM1 model. Addional test statistics on annual basis for $95 \%$ Confidence Interval and at $p=0.05$ Significance levels for the EMI model are available from the author.

Table 2. One-Sample Kolmogorov-Smirnov test run on an Annual basis with the EM2 model, with $p=0.01$ Significance level.

\begin{tabular}{|c|c|c|c|c|c|c|c|c|c|c|c|c|c|c|c|c|}
\hline Debt to Equity (DTE) & 2015 & 2014 & 2013 & 2012 & 2011 & 2010 & 2009 & 2008 & 2007 & 2006 & 2005 & 2004 & 2003 & 2002 & 2001 & 2000 \\
\hline Std. Deviation & .30 & .51 & .37 & .04 & .64 & .71 & 2.98 & 2.05 & 1.32 & .53 & .13 & .32 & .29 & .23 & .22 & .05 \\
\hline Asy & .019 & .003 & .003 & .003 & .000 & .000 & .000 & .000 & .000 & .000 & .003 & .000 & .006 & .000 & .002 & .004 \\
\hline Mont & .026 & .002 & .008 & .004 & .000 & .000 & .000 & .000 & .000 & .000 & .004 & .000 & .006 & .000 & .004 & .008 \\
\hline Equity to Total Assets (ETA) & 2015 & 2014 & 2013 & 2012 & 2011 & 2010 & 2009 & 2008 & 2007 & 2006 & 2005 & 2004 & 2003 & 2002 & 2001 & \\
\hline Std. De & .08 & .09 & .07 & .07 & .15 & .15 & .06 & .03 & .06 & .05 & .02 & .06 & 2.81 & 2.66 & .17 & \\
\hline Kolmo & 1.39 & 1.31 & 1.06 & 1.11 & 1.74 & 1.92 & 1.70 & 1.40 & 1.10 & 1.17 & 1.34 & 0.50 & 2.55 & 2.53 & 1.80 & \\
\hline Asy & .042 & .064 & .207 & .172 & .005 & .001 & .006 & .040 & .177 & .127 & .055 & .967 & .000 & .000 & .003 & \\
\hline Monte Carlo Sig. (2-tailed) & .023 & .065 & .190 & .157 & .007 & .000 & .009 & .021 & .146 & .095 & .056 & .944 & .000 & .000 & .000 & \\
\hline
\end{tabular}


Table 2. Cont.

\begin{tabular}{|c|c|c|c|c|c|c|c|c|c|c|c|c|c|c|c|c|}
\hline Equity to Loans (ETL) & 2015 & 2014 & 2013 & 2012 & 2011 & 2010 & 2009 & 2008 & 2007 & 2006 & 2005 & 2004 & 2003 & 2002 & 2001 & \\
\hline $\mathrm{N}$ & 31 & 29 & 29 & 30 & 29 & 29 & 28 & 28 & 28 & 31 & 31 & 29 & 29 & 32 & 32 & \\
\hline Mean & .63 & -.05 & -.05 & -.05 & -.06 & -.04 & -.04 & -.04 & -.05 & -.05 & -.05 & -.05 & -.07 & -.03 & -.05 & \\
\hline Std. Deviation & 3.78 & .01 & .03 & .04 & .10 & .04 & .07 & .03 & .01 & .01 & .00 & .00 & .10 & .10 & .03 & \\
\hline Kolmogorov-Smirnov Z & 2.99 & 1.48 & 2.08 & 2.13 & 2.64 & 2.67 & 2.42 & 2.44 & 1.62 & 1.56 & 1.13 & 0.93 & 2.76 & 2.78 & 2.05 & \\
\hline Asymp. Sig. (2-tailed) & .000 & .025 & .000 & .000 & .000 & .000 & .000 & .000 & .010 & .015 & .155 & .347 & .000 & .000 & .000 & \\
\hline Monte Carlo Sig. (2-tailed) & .000 & .025 & .000 & .000 & .000 & .000 & .000 & .000 & .004 & .007 & .124 & .306 & .000 & .000 & .000 & \\
\hline Gross Margin Index (GMI) & 2015 & 2014 & 2013 & 2012 & 2011 & 2010 & 2009 & 2008 & 2007 & 2006 & 2005 & 2004 & 2003 & 2002 & 2001 & \\
\hline $\mathrm{N}$ & 39 & 39 & 39 & 36 & 36 & 37 & 35 & 35 & 36 & 37 & 35 & 30 & 30 & 35 & 38 & \\
\hline Mean & .22 & -.12 & -.22 & -.22 & .41 & -.15 & -.39 & -.02 & .08 & .24 & -.64 & .01 & .28 & .40 & .14 & \\
\hline Std. Deviation & .33 & .34 & .42 & .54 & .70 & .48 & .62 & .63 & .77 & .54 & 1.21 & 1.85 & 1.57 & 1.48 & 1.54 & \\
\hline Kolmogorov-Smirnov Z & 0.87 & 0.92 & 1.07 & 1.20 & 1.15 & 0.63 & 1.15 & 0.62 & 1.24 & 1.44 & 1.51 & 1.28 & 1.28 & 1.23 & 1.50 & \\
\hline Asymp. Sig. (2-tailed) & .431 & .365 & .200 & .115 & .143 & .825 & .145 & .831 & .092 & .031 & .021 & .075 & .077 & .097 & .022 & \\
\hline Monte Carlo Sig. (2-tailed) & .358 & .292 & .171 & .095 & .117 & .782 & .114 & .782 & .074 & .019 & .011 & .063 & .063 & .071 & .013 & \\
\hline $\begin{array}{c}\text { Gross Yield on Earning } \\
\text { Assets (GYEA) }\end{array}$ & 2015 & 2014 & 2013 & 2012 & 2011 & 2010 & 2009 & 2008 & 2007 & 2006 & 2005 & 2004 & 2003 & 2002 & 2001 & 2000 \\
\hline $\mathrm{N}$ & 40 & 43 & 41 & 40 & 40 & 40 & 40 & 40 & 39 & 37 & 37 & 38 & 37 & 37 & 40 & 42 \\
\hline Mean & -.03 & -.10 & -.20 & .03 & -.17 & .03 & .11 & -.46 & .38 & -.08 & -.16 & .10 & .65 & .01 & -.02 & -.04 \\
\hline Std. Deviation & .39 & .37 & .47 & .45 & .90 & 1.02 & .64 & 2.37 & 2.42 & .57 & .47 & .53 & .44 & .12 & .05 & .08 \\
\hline Kolmogorov-Smirnov Z & 1.11 & 1.39 & 1.39 & 1.05 & 1.55 & 1.65 & 1.43 & 2.60 & 2.37 & 1.05 & 1.09 & 0.90 & 1.11 & 1.61 & 1.19 & 1.40 \\
\hline Asymp. Sig. (2-tailed) & .171 & .042 & .041 & .224 & .016 & .009 & .033 & .000 & .000 & .225 & .183 & .389 & .173 & .011 & .119 & .039 \\
\hline Monte Carlo Sig. (2-tailed) & .119 & .027 & .017 & .165 & .010 & .006 & .010 & .000 & .000 & .193 & .162 & .350 & .152 & .003 & .070 & .022 \\
\hline
\end{tabular}

\section{Interest (Sales) Receivables} Index (IRI)

\begin{tabular}{rrrrrrrrrrrrrrr}
2015 & 2014 & 2013 & 2012 & 2011 & 2010 & 2009 & 2008 & 2007 & 2006 & 2005 & 2004 & 2003 & 2002 & 2001 \\
\hline 38 & 37 & 37 & 35 & 35 & 36 & 35 & 35 & 35 & 37 & 35 & 29 & 29 & 35 & 38
\end{tabular} $\mathrm{N}$

\begin{tabular}{rrrrrrrrrrrrrrr}
-.04 & .01 & .07 & -.06 & .02 & .06 & -.07 & -.44 & .44 & .05 & .01 & .15 & -.13 & -.03 & -.04 \\
.29 & .18 & .41 & .14 & .25 & .19 & .14 & 2.77 & 2.58 & .22 & .15 & .13 & .13 & .12 & .32 \\
1.92 & 1.13 & 2.22 & 1.21 & 1.41 & 1.35 & 1.19 & 2.77 & 2.74 & 1.10 & 1.06 & 1.12 & 0.89 & 0.72 & 1.74 \\
.001 & .159 & .000 & .106 & .037 & .053 & .116 & .000 & .000 & .179 & .215 & .161 & .407 & .675 & .005 \\
.002 & .137 & .000 & .101 & .040 & .042 & .112 & .000 & .000 & .152 & .205 & .131 & .350 & .656 & .006 \\
\hline
\end{tabular}


Table 2. Cont.

\begin{tabular}{|c|c|c|c|c|c|c|c|c|c|c|c|c|c|c|c|c|c|}
\hline $\begin{array}{c}\text { Loans to Total Assets } \\
\text { (LTA) }\end{array}$ & 2015 & 2014 & 2013 & 2012 & 2011 & 2010 & 2009 & 2008 & 2007 & 2006 & 2005 & 2004 & 2003 & 2002 & & 2001 & 2000 \\
\hline $\mathrm{N}$ & 38 & 33 & 33 & 38 & 37 & 37 & 37 & 38 & 38 & 37 & 37 & 38 & 37 & 37 & 7 & 39 & 42 \\
\hline Mean & .16 & .34 & -.59 & -.26 & -.07 & -.13 & -.09 & .05 & .16 & -.15 & .03 & -.08 & .02 & .25 & & .15 & .17 \\
\hline Std. Deviation & .92 & .85 & 1.03 & 1.00 & .69 & .95 & 1.09 & 1.06 & 1.15 & 1.31 & 1.13 & 1.00 & .83 & 1.07 & & .94 & .62 \\
\hline Kolmogorov-Smirnov Z & 0.50 & 0.59 & 0.77 & 1.10 & 1.20 & 1.18 & 0.96 & 0.89 & 0.74 & 0.55 & 0.76 & 0.56 & 0.67 & 0.79 & & 0.79 & 1.23 \\
\hline Asymp. Sig. (2-tailed) & .962 & .877 & .588 & .176 & .111 & .125 & .314 & .411 & .647 & .922 & .606 & .911 & .766 & .55 & & .554 & .095 \\
\hline $\begin{array}{l}\text { Monte Carlo Sig. (2- } \\
\text { tailed) }\end{array}$ & .941 & .834 & .552 & .133 & .092 & .099 & .265 & .336 & .586 & .899 & .532 & .876 & .711 & .487 & & .512 & .060 \\
\hline Loans to Deposits (LTD) & 2015 & 2014 & 2013 & 2012 & 2011 & 2010 & 2009 & 2008 & 2007 & 2006 & 2005 & 2004 & 2003 & 2002 & & 2001 & 2000 \\
\hline $\mathrm{N}$ & 32 & 31 & 31 & 30 & 30 & 30 & 30 & 29 & 29 & 31 & 31 & 31 & 31 & 3 & & 34 & 35 \\
\hline Mean & .00 & -.66 & .65 & .00 & .00 & .00 & .00 & .00 & .00 & .00 & .00 & .00 & .00 & .00 & & .00 & .00 \\
\hline Std. Deviat & .06 & 2.79 & 2.80 & .03 & .02 & .03 & .01 & .02 & .02 & .01 & .01 & .01 & .00 & .0 & & .01 & .01 \\
\hline rov-Smirnov Z & 1.94 & 2.66 & 2.66 & 2.10 & 1.42 & 1.87 & 1.03 & .88 & 1.26 & 1.64 & 1.53 & .98 & 1.33 & 1.7 & & 1.69 & 2.24 \\
\hline Asymp. Sig. (2-tailed) & .001 & .000 & .000 & .000 & .034 & .002 & .234 & .418 & .080 & .009 & .018 & .290 & .056 & .00 & & .007 & .000 \\
\hline $\begin{array}{l}\text { Monte Carlo Sig. (2- } \\
\text { tailed) }\end{array}$ & .000 & .000 & .000 & .000 & .028 & .002 & .234 & .405 & .056 & .002 & .022 & .280 & .065 & .00 & & .008 & .000 \\
\hline Net interest Margin (NIM) & 2015 & 2014 & 2013 & 2012 & 2011 & 2010 & 2009 & 2008 & 2007 & 2006 & 2005 & 2004 & 2003 & 2002 & 2001 & & \\
\hline $\mathrm{N}$ & 39 & 38 & 38 & 36 & 36 & 37 & 35 & 35 & 36 & 37 & 35 & 30 & 30 & 35 & 38 & & \\
\hline $\mathrm{Me}$ & -.06 & -.09 & -.04 & $4-.37$ & .31 & -.06 & -.19 & -.11 & .17 & -.14 & -.08 & -.22 & 1.02 & .02 & -.01 & & \\
\hline Std. & .83 & .90 & .49 & 1.55 & 1.65 & .29 & 1.05 & 1.11 & 1.11 & .30 & 1.17 & 1.28 & .77 & .30 & .24 & & \\
\hline Kolmogorov-Smirnov Z & 1.80 & 1.44 & 0.74 & +1.48 & 1.52 & 0.65 & 1.14 & 1.09 & 0.86 & 1.63 & 1.17 & 0.76 & 0.93 & 1.49 & 1.70 & & \\
\hline Asymp. Sig. (2-tailed) & .003 & .031 & .636 & 5.024 & .019 & .800 & .149 & .184 & .458 & .010 & .129 & .605 & .348 & .024 & .006 & & \\
\hline Monte Carlo Sig. (2-tailed) & .000 & .030 & .568 & 3.024 & .019 & .763 & .121 & .155 & .436 & .009 & .105 & .589 & .342 & .024 & .004 & & \\
\hline Profit Margin (PATM) & 2015 & 2014 & 2013 & 2012 & 2011 & 2010 & 2009 & 2008 & 2007 & 2006 & 2005 & 2004 & 2003 & 2002 & 2001 & 2000 & \\
\hline $\mathrm{N}$ & 40 & 43 & 41 & 41 & 38 & 38 & 39 & 40 & 40 & 37 & 37 & 34 & 34 & 34 & 39 & 41 & 1 \\
\hline Mea & .15 & -.32 & .03 & -.03 & -.10 & -.13 & -.07 & -.05 & -.15 & -.14 & -.24 & .19 & .34 & .00 & -.05 & .61 & \\
\hline Std. De & 1.10 & .96 & .74 & .33 & .50 & .49 & .60 & .85 & .87 & .40 & 1.39 & 1.17 & .61 & 1.22 & 1.54 & 1.66 & \\
\hline Kolmogorov-Smirnov Z & 1.11 & 0.94 & 0.92 & 1.42 & 0.72 & 1.13 & 1.40 & 1.81 & 2.22 & 1.32 & 2.53 & 2.23 & 0.91 & 0.75 & 0.81 & 1.59 & \\
\hline Asymp. Sig. (2-tailed) & .171 & .340 & .365 & .035 & .679 & .156 & .039 & .003 & .000 & .060 & .000 & .000 & .380 & .634 & .532 & .013 & \\
\hline Monte Carlo Sig. (2-tailed) & .177 & .310 & .362 & .023 & .628 & .141 & .026 & .000 & .000 & .047 & .000 & .000 & .333 & .593 & .494 & .005 & \\
\hline
\end{tabular}


Table 2. Cont.

\begin{tabular}{|c|c|c|c|c|c|c|c|c|c|c|c|c|c|c|c|c|}
\hline Return on Asset (ROA) & 2015 & 2014 & 2013 & 2012 & 2011 & 2010 & 2009 & 2008 & 2007 & 2006 & 2005 & 2004 & 2003 & 2002 & 2001 & \\
\hline $\mathrm{N}$ & 39 & 38 & 38 & 37 & 37 & 37 & 35 & 35 & 37 & 37 & 35 & 31 & 31 & 35 & 37 & \\
\hline Mean & .38 & -.48 & .30 & .00 & -.01 & -.24 & -.05 & .09 & -.24 & -.09 & .04 & .02 & .38 & .00 & -.05 & \\
\hline Std. Deviation & 1.07 & 1.50 & 1.25 & 1.36 & 1.12 & .81 & .70 & .79 & 1.44 & .23 & .62 & .61 & .92 & .82 & .33 & \\
\hline Kolmogorov-Smirnov Z & 1.23 & 1.11 & 1.26 & 0.92 & 0.94 & 1.45 & 1.26 & 1.32 & 1.90 & 1.27 & 0.77 & 0.76 & 1.69 & 1.85 & 1.52 & \\
\hline Asymp. Sig. (2-tailed) & .097 & .173 & .085 & .359 & .344 & .030 & .083 & .061 & .002 & .080 & .598 & .609 & .007 & .002 & .020 & \\
\hline Monte Carlo Sig. (2-tailed) & .067 & .173 & .085 & .317 & .297 & .020 & .091 & .061 & .000 & .067 & .570 & .596 & .002 & .000 & .019 & \\
\hline Return on Equity (ROE) & 2015 & 2014 & 2013 & 2012 & 2011 & 2010 & 2009 & 2008 & 2007 & 2006 & 2005 & 2004 & 2003 & 2002 & 2001 & \\
\hline $\mathrm{N}$ & 31 & 29 & 29 & 32 & 30 & 28 & 27 & 27 & 29 & 32 & 31 & 29 & 29 & 32 & 33 & \\
\hline Mean & .09 & -.08 & -.02 & .03 & .05 & -.02 & -.10 & .17 & -.24 & .04 & .01 & .04 & .03 & .03 & -.03 & \\
\hline Std. Deviation & 44 & .35 & .19 & 48 & 1.06 & 1.07 & .32 & 2.57 & 2.52 & .43 & .13 & 20 & .16 & .18 & .24 & \\
\hline Kolmogorov-Smirnov Z & 2.01 & 1.65 & 1.24 & 1.77 & 2.01 & 1.91 & 1.86 & 2.36 & 2.13 & 2.29 & 1.48 & 1.86 & 1.53 & 2.33 & 2.57 & \\
\hline Asymp. Sig. (2-tailed) & .001 & .009 & .092 & .004 & .001 & .001 & .002 & .000 & .000 & .000 & .025 & .002 & .018 & .000 & .000 & \\
\hline Monte Carlo Sig. & .000 & .007 & .076 & .002 & .000 & .000 & .002 & .000 & .000 & .000 & .018 & .002 & .013 & .000 & .000 & \\
\hline Rate Paid on Fu & 2015 & 2014 & 2013 & 2012 & 2011 & 2010 & 2009 & 2008 & 2007 & 2006 & 2005 & 2004 & 2003 & 2002 & 2001 & 2000 \\
\hline $\mathrm{N}$ & 37 & 39 & 38 & 37 & 37 & 37 & 38 & 40 & 38 & 37 & 37 & 38 & 35 & 35 & 39 & 41 \\
\hline Mean & .00 & -.01 & -.01 & .00 & .00 & -.02 & -.02 & -.01 & -.42 & .46 & -.01 & .01 & .04 & .00 & .00 & .00 \\
\hline Std. Deviation & .02 & .02 & .03 & .03 & .02 & .04 & .05 & .24 & 2.82 & 2.84 & .03 & .04 & .03 & .01 & .00 & .01 \\
\hline Kolmo & 0.85 & 0.68 & 0.59 & 0.93 & 0.62 & 1.47 & 0.82 & 2.72 & 3.22 & 3.23 & 0.90 & 1.03 & 1.10 & 1.62 & 0.98 & 1.51 \\
\hline Sig. (2-tailed) & .461 & .747 & .883 & .356 & .840 & .027 & .519 & .000 & .000 & .000 & .392 & .236 & .180 & .010 & .295 & .021 \\
\hline Monte & .388 & .695 & .833 & .292 & .781 & .022 & .469 & .000 & .000 & .000 & .328 & .207 & .181 & .013 & .272 & .020 \\
\hline Sales Gro & 2015 & 2014 & 2013 & 2012 & 2011 & 2010 & 2009 & 2008 & 2007 & 2006 & 2005 & 2004 & 2003 & 2002 & 2001 & \\
\hline $\mathrm{N}$ & 40 & 38 & 38 & 36 & 36 & 37 & 36 & 35 & 35 & 37 & 37 & 30 & 30 & 35 & 38 & \\
\hline Mean & .00 & .00 & -.02 & -.02 & .04 & -.09 & -.01 & -.04 & -.20 & .30 & -.06 & -2.62 & 2.45 & .16 & .07 & \\
\hline Std. Dev & .10 & .10 & .09 & .09 & .08 & .23 & .25 & .50 & .42 & .26 & .11 & 1.29 & 1.43 & .56 & .29 & \\
\hline Kolmogorov-Smirnov Z & 1.35 & 1.08 & 1.15 & 1.32 & 1.47 & 2.10 & 1.59 & 2.40 & 1.61 & 1.16 & 1.07 & 0.68 & 0.83 & 1.86 & 1.60 & \\
\hline Asymp. Sig. (2-tailed) & .052 & .196 & .145 & .060 & .026 & .000 & .013 & .000 & .011 & .134 & .202 & .748 & .498 & .002 & .012 & \\
\hline Monte Carlo Sig. (2-tailed) & .030 & .154 & .110 & .041 & .015 & .000 & .004 & .000 & .002 & .095 & .160 & .706 & .437 & .000 & .004 & \\
\hline
\end{tabular}

Notes: Statistically significant years with 99\% Confidence Interval and with $p=0.01$ Significance level for DTE are: 2000-2014; for ETA: 2001-2003, 2009-2011; for ETL: 200120013, 2007-2013, 2015, and addditionally 2006 for Monte Carlo Sig. only; for GMI are statistically insignificant; for GYEA significant years are: 2007-2008, 2010, and 2002, 2009, 2011 for Monet Carlo Sig. only; for IRI: 2001, 2007-2008, 2013, 2015; for LTA are statistically insignificant; for LTD significant years are: 2000-2002, 2006, 2010, 20122015; for NIM: 2001-2002, 2006, 2015; for PATM are: 2004-2005, 2007-2008 and 2000 for Monte Carlo only; ROA: 2002-2003, 2007; for ROE: 2001-2002, 2004, 2006-2012, 2014-2015; for RPF are: 2006-2008, and 2002 for Asump. Sig. only; significant years for SGI are: 2002, 2008, 2010, and 2001, 2007, and 2009 for Monte Carlo Sig. only. 
The EM2 model in Table 2, for the same tested ratios, shows almost identical statistically significant results as for the EMI model in Table 1. This would suggests, that managers in order to outplay the strict financial rules, engage in EM by manipulating different areas of financial statements with different techniques, see e.g., Beretka (2016, 22-24), and at their discretion, in years / periods of their choosing. The results suggest that the hypotheses $\boldsymbol{H O}_{(a)}$ : and $\boldsymbol{H O}_{(b)}$ : 'may' be rejected for the statistically significant years. Only GMI, LTA and NIM ratios show overall statistically insignificant results for each financial year for both EMI and EM2 models; therefore, the hypothesis $\boldsymbol{H} \boldsymbol{O}_{(b)}$ : holds for GMI, LTA and NIM ratios. Statistical outputs for the 14 ratios for the EM2 model for both Confidence Interval and Significance levels are very similar to EM1 model. Addional test statistics on annual basis for 95\% Confidence Interval and at $p=0.05$ Significance levels for the EM2 model are available from the author.

\section{The 2008 Financial Crisis}

This paper additionally investigates evidence of EM 'Prior to' and 'After' the 2008 financial crisis. Hypothesis $\boldsymbol{H O}_{(\boldsymbol{b})}$ : was tested with the K-S test statistics by applying the EMI and the EM2 models with the 14 bank specific ratios. In order to analyse 'Prior to' and 'After' 2008 financial crisis, 'Prior to' 2008 years were set from 2001 to 2007, whereas 'After' the financial crisis years are from 2008 to 2014. As it can be seen in Table 1, only Gross Margin Index (GMI), Loans to Total Assets (LTA) at $p=0.01$ Significance Levels have statistically insignificant outputs. Same is true for tests run with the EM2 model, statistically insignificant outputs at $p=0.01$ are for GMI and LTA ratio and at $p=0.05$ LTA ratio only. Statistical test outputs for EM1 and EM2 models for significance levels of $p=0.05$ are available from the author. The insignificance of the outputs for GMI ratio suggests that it is less likely that it is being manipulated. The statistical insignificance of LTA suggests that there are no outstanding loans and that the liquidity of the firms is well managed. This was not the case, as the LTA statistical outputs do not show the reality of the credit institutions' operations, which had poor liquidity and high lending, as the DTE and ETL statistical results point out. Evidence bearing out that the LTA statistical outputs are false is shown in the lending practices to Households, Consumer and Corporate Sectors in Figures 3.1, 3.1, 3.3, Beretka (2016, 106-110). Lending was in significant increase from 2003 within all three sectors with its peak in 2009 that prompted illiquidity of the lending institutions and forced bailouts of some credit institutions trading in Hungary. From 2009, astonishingly, lending maintained its level for all three sectors. In summary, it can be argued, given by the evidence from Tables 1 and 2, that managers engaged in EM 'Prior to' the 2008 financial crisis possibly to maintain parent companies' profit expectations, thus kept engaging in EM even 'After' the 2008 financial crisis. Tests statistics show clear evidence that hypothesis $\boldsymbol{H O}_{(b)}$ : 'may' be rejected.

\section{Benchmark Analysis}

Earlier evidence suggests that research papers seldom test Benchmark when investigating EM. Tables 3 and 4 present Descriptive Statistics output for Benchmark and for Base ratios. Only a handful of papers argue, see e.g., Dechow, Ge and Schrand (2010, 351), in favour of including Benchmark analysis with test statistics and/or with a combination of histograms, see e.g., Beretka (2016, 189-199). By comparing each ratio between the Base and Benchmark outputs in Tables 3 and 4 , it can be concluded that results differ, thus indicate that there is a significant difference for Mean and S.D. are for DTE and for LTD. Observing only S.D. for ETL, PATM and ROE we may conclude that outputs show high values, suggesting that they are widely spread and are less reliable; whereas high Means for DTE, LTD suggest that they are spread away from the central point. Skewness and Kurtosis are positive and negative for both Benchmark and Base ratios outputs. Negative Skewness for ROA and ROE in the Benchmark output suggest that the values are on the right of the distribution, whereas positive values are on the left. Positive Kurtosis for benchmark output in Table 3 are positive thus suggesting "ponty and heavily-tailed distribution, 
Table 3. Benchmark Descriptive Statistics

\begin{tabular}{|c|c|c|c|c|c|c|c|c|c|c|c|}
\hline & $\mathrm{N}$ & Min & Max & Mean & $\begin{array}{l}\text { Mean } \\
\text { Std. } \\
\text { Error }\end{array}$ & S.D. & $\begin{array}{c}\text { S.D. } \\
\text { Variance }\end{array}$ & $\begin{array}{l}\text { Skew- } \\
\text { ness }\end{array}$ & $\begin{array}{l}\text { Skew } \\
\text { Std. } \\
\text { Error }\end{array}$ & $\begin{array}{l}\text { Kurt- } \\
\text { osis }\end{array}$ & $\begin{array}{l}\text { Kurt } \\
\text { Std. } \\
\text { Err. }\end{array}$ \\
\hline DTE & 17 & 419.4 & 6023 & 1608 & 308.4 & 1271 & 1617539 & 2.87 & 0.55 & 9.78 & 1.06 \\
\hline ETL & 16 & 0.02 & 9.23 & 0.68 & 0.57 & 2.28 & 5.21 & 3.99 & 0.56 & 15.9 & 1.09 \\
\hline LTD & 17 & 1.41 & 1807 & 228 & 107 & 444 & 197111 & 3.18 & 0.55 & 11.1 & 1.06 \\
\hline LTA & 17 & 0.18 & 1.85 & 0.59 & 0.08 & 0.34 & 0.12 & 3.45 & 0.55 & 13.7 & 1.06 \\
\hline GYEA & 17 & 0.07 & 0.96 & 0.49 & 0.06 & 0.27 & 0.07 & -0.4 & 0.55 & -0.67 & 1.06 \\
\hline RPF & 17 & 0.00 & 0.53 & 0.06 & 0.03 & 0.12 & 0.02 & 3.85 & 0.55 & 15.38 & 1.06 \\
\hline SGI & 16 & 0.87 & 12.5 & 2.01 & 0.71 & 2.84 & 8.07 & 3.82 & 0.56 & 14.93 & 1.09 \\
\hline IRI & 16 & 0.19 & 3.04 & 1.18 & 0.14 & 0.57 & 0.33 & 2.18 & 0.56 & 8.40 & 1.09 \\
\hline GMI & 16 & 0.78 & 1.25 & 0.98 & 0.03 & 0.12 & 0.01 & 0.39 & 0.56 & 0.10 & 1.09 \\
\hline NIM & 16 & 0.05 & 0.40 & 0.29 & 0.03 & 0.12 & 0.01 & -1.3 & 0.56 & 0.51 & 1.09 \\
\hline PATM & 17 & -3.54 & 8.87 & 0.15 & 0.60 & 2.47 & 6.08 & 2.85 & 0.55 & 11.13 & 1.06 \\
\hline ROE & 16 & -20.1 & 23.5 & -0.5 & 2.99 & 11.9 & 142.9 & 0.21 & 0.56 & -0.08 & 1.09 \\
\hline ROA & 16 & -0.02 & 0.02 & 0.00 & 0.00 & 0.01 & 0.00 & -0.3 & 0.56 & -0.33 & 1.09 \\
\hline ETA & 16 & 0.01 & 0.22 & 0.03 & 0.01 & 0.05 & 0.00 & 3.93 & 0.56 & 15.60 & 1.09 \\
\hline
\end{tabular}

Notes: Tests ran for the period 1999 - 2015.

while negative values suggest 'flat' distribution, see e.g., Field $(2009,138)$. However, Benchmark results should be read with caution, due to the specifics of the Hungarian Accoiunting Standards and the lack of quality research availability. Table 3 Benchmark outputs were calculated from the same sample as the Base ratios statistics. Despite the lack of research material, Benchmark comparisons should not be excluded from research.

Table 4. Descriptive Statistics

\begin{tabular}{|c|c|c|c|c|c|c|c|c|c|c|c|}
\hline & $\mathrm{N}$ & Min & $\operatorname{Max}$ & Mean & $\begin{array}{c}\text { Mean } \\
\text { Std. } \\
\text { Error }\end{array}$ & S.D. & S.D. Var. & $\begin{array}{c}\text { Skew- } \\
\text { ness }\end{array}$ & $\begin{array}{l}\text { Skew } \\
\text { Std. } \\
\text { Err. }\end{array}$ & $\begin{array}{l}\text { Kur- } \\
\text { tosis }\end{array}$ & $\begin{array}{l}\text { Kur } \\
\text { Std. } \\
\text { Err. }\end{array}$ \\
\hline DTE & 568 & 0.00 & 152260 & 1566 & 315 & 7524 & 56610025 & 15.4 & 0.10 & 290 & 0.20 \\
\hline ETL & 515 & 0.00 & 349.6 & 0.78 & 0.68 & 15.42 & 237.66 & 22.6 & 0.11 & 513 & 0.21 \\
\hline LTD & 579 & 0.00 & 72149 & 257 & 134 & 3225 & 10403539 & 19.8 & 0.10 & 432 & 0.20 \\
\hline LTA & 669 & 0.00 & 53.06 & 0.60 & 0.08 & 2.05 & 4.20 & 25.1 & 0.09 & 644 & 0.19 \\
\hline GYEA & 682 & 0.01 & 13.63 & 0.49 & 0.02 & 0.65 & 0.42 & 12.9 & 0.09 & 250 & 0.19 \\
\hline RPF & 664 & -0.0 & 19.12 & 0.07 & 0.03 & 0.74 & 0.55 & 25.3 & 0.09 & 650 & 0.19 \\
\hline SGI & 592 & 0.00 & 25.56 & 1.89 & 0.12 & 2.97 & 8.85 & 4.5 & 0.10 & 22.8 & 0.20 \\
\hline IRI & 584 & 0.00 & 84.31 & 1.24 & 0.15 & 3.58 & 12.85 & 21.5 & 0.10 & 497 & 0.20 \\
\hline GMI & 588 & -2.3 & 5.38 & 0.99 & 0.02 & 0.48 & 0.23 & 1.0 & 0.10 & 29.6 & 0.20 \\
\hline NIM & 591 & -0.0 & 3.30 & 0.29 & 0.01 & 0.31 & 0.09 & 3.5 & 0.10 & 20.2 & 0.20 \\
\hline PATM & 673 & -118 & 324.4 & 0.06 & 0.52 & 13.47 & 181.4 & 19.8 & 0.09 & 511 & 0.19 \\
\hline $\mathrm{ROE}$ & 502 & -2062 & 1098 & -0.4 & 5.46 & 122.4 & 14984 & -8.9 & 0.11 & 179 & 0.22 \\
\hline ROA & 596 & -0.3 & 0.18 & 0.00 & 0.00 & 0.04 & 0.00 & -2.4 & 0.10 & 16.6 & 0.20 \\
\hline ETA & 502 & 0.00 & 5.89 & 0.03 & 0.01 & 0.26 & 0.07 & 21.8 & 0.11 & 486 & 0.22 \\
\hline
\end{tabular}

Notes: Descriptive Statistics for Base Ratios for the period 1999 - 2015. No modelling was performed. 


\section{Discussion}

The Distribution of Ratios research approach applies a technique to use all possible computable variables from the financial statements, thus ratios that do not contain assets and/or liabilities, as component in their formula, it eliminates the reversal accrual impact and increases the credibility and power of the statistical test results in comparison to accrual testing models. By rejecting Hypotheses $\boldsymbol{H O}_{(a)}$ : and $\boldsymbol{H} \boldsymbol{0}_{(b)}$ : for ratios that comprise current assets or total assets (i.e. ETA, LTA, ROA), or current / long term liabilities (i.e. DTE, ETL, LTA, LTD), which may have influenced the test statistics due to reversal accruals, a component of assets and/or liabilities in the balance sheet, this study could have made a Type I error.

\section{Conclusion}

This paper examined an alternative research approach, the Distribution of the Ratios method in search of evidence of Earnings Management of annual accounts of the Hungarian credit institutions (banks). Test outputs run on an annual basis with the EM1 and the EM2 models for each ratio present more details, specifically highlighting that evidence of Earnings Management, thus rejecting $\boldsymbol{H O}_{(a)}$ : Furthermore, evidence for EM1 and EM2 models present an opportunistic approach by managers who manipulate accounting entries at their discretion in different time frames and in all areas of the financial statements, thus $\boldsymbol{H 0}_{(b)}$ : is rejected. Benchmark analysis was performed with the same sample as the base ratios data. Due to lack of benchmark material for financials and for credit institutions in Hungary, the results should be read with caution. However, when industry benchmark data is available, it should be used for comparison. This paper also investigated the 2008 financial crisis, and concluded that 'Prior to' and 'After' the 2008 financial crisis there is evidence of Earnings Management, thus $\boldsymbol{H 0}_{(\boldsymbol{b})}$ : is rejected. The EM1 and EM2 models test results on annual basis show statistical significant results for $99 \%$ confidence and $p=0.01$ significant levels for all 14 tested ratios. Test results for 14 ratios for the EM1 and EM2 models tested with all sample and with annual data of $95 \%$ confidence interval and $p=$ 0.05 significance level are statistically significant and they are available from the author.

\section{References}

Baber, W. R., Kang, S.H. and Li Y. 2011. "Modelling discretionary accrual reversal and the balance sheet as an earnings management constraint." Accounting Review 86(4): 1189-1212.

Beatty, A., Chamberlain, A. and Magliolo, J. 1995. "Managing Financial Reports of Commercial Banks: The Influence of Taxes, Regulatory Capital, and Earnings.” Journal of Accounting Research 33 (2): 231-261.

Beatty, A., Ke, B. and Petroni, K. 2002. "Earnings Management to Avoid Earnings declines across Publicly and Privately Held Banks." The Accounting Review 77(3): 547-570.

Beneish, M.D. 1999. "The Detection of Earnings Manipulation.” Financial Analyst Journal 55(5) (Sept. - Oct): 24-36.

Beretka, E. 2016. "Earnings Management: A Study of Credit Institutions in Hungary 1999-2012." [online] Available from British Library: http://ethos.bl.uk/OrderDetails.do?did=1\&uin=uk.bl.ethos.707517.

Burgstahler, D. and Chuck, E. 2013. "What Have We Learned About Earnings Management? Correcting Disinformation about Discontinuities." Working Paper Series, June. Available at SSRN: http://ssrn.com/abstract=1866008 or http://dx.doi.org/10.2139/ssrn.1866008.

Burgstahler, D. and Dichev I. 1997. "Earnings management to avoid earnings decreases and losses." Journal of Accounting and Economics 24: 99-126.

Crump, K. S. and Teeguarden, J. G., 2009. "Benchmark calculations from summarised data: an example.” Environmental and Ecological Statistics 16(1): 13-24.

Dattakumar, R. and Jagadeesh, R. 2003. "A review of literature on benchmarking. Benchmarking." An International Journal 10(3):176-209.

Dechow, P.M., Ge, W. and Schrand, C. 2010. "Understanding earnings quality: A review of the proxies, their determinants and their consequences." Journal of Accounting \& Economics 50: 344-401.

Dechow, P.M., Hutton, A.P., Kim, J.H. and Sloan, R.G. 2012. "Detecting Earnings Management: A New Approach." Journal of Accounting Research, Vol. 50, No. 2, May.

Degeorge, F., Patel, J. and Zeckhauser, R. 1999. "Earnings Management to Exceed Threshold.” Journal of Business. 72(1): $1-33$.

Field, A. 2009. “Discovering Statistics Using SPSS.” $3^{\text {rd }}$ Edition, SAGE Publications Ltd, ISBN 978-1-84787-906-6.

Gore, P., Pope, P. and Singh A.K. 2007. "Earnings management and the distribution of earnings relative to targets: UK evidence." Accounting and Business Research 37 (2): 123-149. 
Habib, A. 2007. "Benchmark Beating' as Evidence of Earnings Management: Review of the Literature”. Working Paper Series, Available at: https://ssrn.com/abstract $=873939$.

Kent, R. and Routledge, J. 2015. "Use of benchmarks in predicting earnings management?" Accounting and Finance 57: 122.

Lilliefors, H. 1967. "On the Kolmogorov-Smirnov Test for Normality with Mean and Variance Unknown." Journal of the American Statistical Association 62(318): 399-402.

Massey, F. 1951. "The Kolmogorov-Smirnov Test for Goodness of Fit." Journal of the American Statistical Association 46(253): 68-78.

Shen, C.H. and Chih, H.L. 2005. "Investor protection, prospect theory and earnings management: An international comparison of the banking industry.".Journal of Banking \& Finance 29: 2675-2697.

Sun, L. and Rath, S. 2012. "Pre Managed Earnings Benchmarks and Earnings Management of Australian Firms." Australasian Accounting Business and Finance Journal 6(1): 29-56. The Central Bank of Hungary, [online] https://www.mnb.hu/en/the-central-bank.

Yasin, M. M. 2002. "The theory and practice of benchmarking: then and now." Benchmarking. An International Journal 9(3): 217-243. 